\section{PTU-074 LOW-DOSE THIOPURINE AND ALLOPURINOL CO-THERAPY RESULTS IN SIGNIFICANT COST SAVINGS AT A DISTRICT GENERAL HOSPITAL}

doi:10.1136/gutjnl-2013-304907.166

\begin{abstract}
1."H E Johnson, ${ }^{2} \mathrm{H}$ M Dewhurst, 'S A Weaver, 'S D McLaughlin. 'Gastroenterology;
\end{abstract} 2Research, Royal Bournemouth Hospital, Bournemouth, UK

Introduction Thiopurines are used for maintenance of remission in IBD. In England and Wales biologics are approved by NICE (National institute for health and clinical excellence) for Crohn's disease (CD) but not ulcerative colitis. Adalimumab is recommended in preference to infliximab in patients over $65 \mathrm{~kg}$ due to cost. Published data report $>50 \%$ of patients stop thiopurines due to theraputic failure, hepatitis or side effects. In this situation most UK clinicians start biologics in CD patients. This has significant cost implications. An alternative treatment strategy is low dose thiopurine and allopurinol (LDTA) co-therapy which is effective in most patients who fail standard dose thiopurines. Some patients require liquid thiopurine to achieve the correct (low) dose -this formulation is significantly more costly than tablets. We report the annual cost savings from adopting this strategy at our centre.

Methods We maintain a prospective IBD database. Patients with CD treated with LDTA in preference to biologic therapy were identified. The annual drug costs of their treatment with LDTA compared with biologic therapy (adalimumab for patients over $65 \mathrm{~kg}$, infliximab for patients $<65 \mathrm{~kg}$ ) were calculated including the cost for the formulation of thiopurine used (liquid/capsule/tablets) and the dose prescribed. Costs of attending the day unit for an infusion were not included.

Results 17 CD patients who failed standard thiopurine and were eligible for biologics were identified over a 1 year period (Sept 2011-Sept 2012). Of these 4 (24\%) failed LDTA and progressed to biologics, $13(76 \%)$ entered a sustained clinical remission. Mean weight of patients $=77.3 \mathrm{~kg}$ (range: $53.5-105), 6(46 \%)$ patients required a liquid thiopurine. Mean calculated costs were: thiopurine $£ 451.95$ (range: $£ 48.48-£ 1345.44$ ). biologic: $£ 11,331$ (range: $£ 10,560-£ 16,081$ ). Mean cost saving per patient: $£ 10,879$ (range: $£ 9,215-£ 15,146)$. Total cost saving: £141,427.

Conclusion We have previously reported that low dose thiopurine and allopurinol co-therapy is safe and effective.

In the present study we have identified significant annual cost savings can be made when this treatment strategy is used to prevent escalation to biologics. These cost savings are likely to be even more significant in the long term since a significant proportion of patients treated with biologic therapy require dose escalation. We believe adopting this strategy more widely could lead to significant health-care savings.

Disclosure of Interest None Declared

\section{PTU-075 LONG TERM OUTCOME OF AZATHIOPRINE THERAPY IN 353 CONSECUTIVE IBD PATIENTS}

doi:10.1136/gutjnl-2013-304907.167

1."H E Johnson, ${ }^{2} \mathrm{~K}$ Smith, 'N Jarrett, 'S D McLaughlin, 'S A Weaver. 'Gastroenterology, Royal Bournemouth Hospital, Bournemouth; 'astroenterology, Yeovil District Hospital, Yeovil, UK

Introduction Thiopurines are the mainstay of therapy in Inflammatory Bowel Disease (IBD). Azathioprine (AZA) and Mercaptopurine are very effective at maintaining remission but have a wide range of side effects which can limit their use as long term maintenance therapy. To assess how effective AZA was in IBD, and what its limitations were, the outcome of 353 consecutive IBD patients started on AZA with at least onw year follow up was assessed.

Methods Since 2005 all patients started on AZA for IBD have been recorded and monitored. These data were then used to assess the outcomes of patients where there had been at least one year of follow up. Outcomes recorded were whether AZA was still being taken or not. If still being taken information about the disease activity was recorded. If AZA therapy had been discontinued then the reason for this was recorded and subsequent therapeutic interventions noted.

Results 353 patients had started AZA and had at least one year of follow up. TPMT status was checked in all patients. Dosing was as follows: low TPMT; $50 \mathrm{mg}$ and increased as tolerated. Normal TPMT; 2-2.5mg/kg.

Of the 353 patients, 204 had Crohn's disease (CD), 141 had Ulcerative Colitis and 8 had IBD-unclassified. The male:female ratio was 184:169 (52.1\% male). Age range was 16-86 years (mean; 46). 322/353 (91\%) remain under follow up.

$127(36 \%)$ of patients stopped taking AZA at one year. After six years 152 (43.1\%) remained on AZA, 182 (51.6\%) had stopped and in $19(5 \%)$ the outcome was unknown. Nausea and myalgia were the main reasons for stopping AZA. 40(11.3\%) patients developed hepatitis (ALT rise > 2xULN), 6 (1.7\%) developed myelosuppression and 7 (2\%) developed pancreatitis (consistent clinical presentation and raised amylase). Of the 182 patients who stopped AZA, 67 (37.8\%) had an escalation of therapy - 20 started methotrexate, 18 started biologics and 29 underwent surgery. Of the 152 who continued AZA, 138 (90.8\%) were in a clinical remission based on clinical assessment supported by normal C-reactive protein in 126 (91.3\%), Harvey Bradshaw Index in those with CD $55(40 \%)$ patients and endoscopic findings in 22 (15.9\%). 112 (73.6\%) patients had blood monitoring (FBC and LFTs) at least quarterly and 147 (96.7\%) at bi-annually.

Conclusion For such an important drug in IBD management a significant number of patients stop AZA due to side effects. This study highlights these so that patients can be accurately informed. It also highlights that AZA when tolerated is a very effective maintenance medication. Published data from our own and other units suggest that low-dose AZA in combination with allopurinol reduces side effects and increases tolerability and may make AZA a more effective longterm maintenance agent.

Disclosure of Interest None Declared

\section{PTU-076 DIAGNOSTIC POTENTIAL OF VOLATILE ORGANIC COMPOUNDS AS FAECAL BIOMARKERS IN INFLAMMATORY BOWEL DISEASE}

doi:10.1136/gutjnl-2013-304907.168

1."H Jayasena, ${ }^{2 \top}$ Khalid, ${ }^{3} \mathrm{C}$ S Probert. ' Gastroenterology, Bristol Royal Infirmary, Bristol; ${ }^{2}$ University of Liverpool, Liverpool, UK; ${ }^{3}$ Institute of Tanslational Medicine, University of Liverpool, Liverpool, UK

Introduction VOCs can be utilised as non invasive biomarkers for gastrointestinal diseases such as IBD, as changes in VOCs reflect internal metabolical and pathological processes.

Methods Patients were recruited from outpatients with proven Crohn's disease(CD $N=41$ ), ulcerative colitis(UC $N=49$ ), IBS $(N=30)$ and healthy volunteers $(N=47)$. Disease activity was recorded using Harvey-Bradshaw index $(\mathrm{HBI})$ in $\mathrm{CD}$ and simple clinical colitis activity index(SCCAI) in UC. Faecal headspace gas was sampled with SPME and transferred to GC-MS for VOC identification. Statistical analysis was performed on presence or absence and peak area of VOCs.

\section{Results}

